Working Paper/Document de travail 2014-33

\title{
Competition in the Cryptocurrency Market
}

\author{
by Neil Gandal and Hanna Hałaburda
}


Bank of Canada Working Paper 2014-33

August 2014

Revised September 2014

\title{
Competition in the Cryptocurrency Market
}

\author{
by \\ Neil Gandal ${ }^{1}$ and Hanna Hałaburda ${ }^{2}$ \\ ${ }^{1}$ Tel Aviv University and CEPR \\ gandal@post.tau.ac.il \\ ${ }^{2}$ Currency Department \\ Bank of Canada \\ Ottawa, Ontario, Canada K1A OG9 \\ and \\ CESifo and INE PAN \\ hhalaburda@gmail.com
}

Bank of Canada working papers are theoretical or empirical works-in-progress on subjects in economics and finance. The views expressed in this paper are those of the authors. No responsibility for them should be attributed to the Bank of Canada. 


\section{Acknowledgements}

We are grateful to Yaniv Friedensohn and Chris Henry for invaluable research assistance. We thank Jonathan Chiu, Geoffrey Dunbar, Ben Fung, Raffaella Giacomini, Scott Hendry, Lukasz Pomorski, Miguel Molico, Gerald Stuber, Russell Wong, and three anonymous referees for very helpful comments and suggestions. 


\begin{abstract}
We analyze how network effects affect competition in the nascent cryptocurrency market. We do so by examining the changes over time in exchange rate data among cryptocurrencies. Specifically, we look at two aspects: (1) competition among different currencies, and (2) competition among exchanges where those currencies are traded. Our data suggest that the winner-take-all effect is dominant early in the market. During this period, when Bitcoin becomes more valuable against the U.S. dollar, it also becomes more valuable against other cryptocurrencies. This trend is reversed in the later period. The data in the later period are consistent with the use of cryptocurrencies as financial assets (popularized by Bitcoin), and not consistent with “winner-take-all” dynamics.
\end{abstract}

JEL classification: $L 1$

Bank classification: E-money

\title{
Résumé
}

Les auteurs analysent l'incidence des effets de réseau sur la concurrence au sein du jeune marché des cryptomonnaies. Pour ce faire, ils examinent l'évolution des taux de change entre les cryptomonnaies au fil du temps. Plus particulièrement, ils étudient: 1) la concurrence entre diverses cryptomonnaies et 2) la concurrence entre les plateformes où elles s'échangent. Dans une première phase, les données évoquent le phénomène classique de concentration autour d'un acteur dominant. Au cours de cette période, lorsque le bitcoin gagne de la valeur par rapport au dollar américain, il s’apprécie également vis-à-vis des autres cryptomonnaies. Durant la seconde phase observée, cette tendance s’inverse. Les données cadrent alors avec l'emploi des cryptomonnaies (que le bitcoin a banalisé) à titre d'actifs financiers, et non avec une dynamique de marché favorisant l'hégémonie.

Classification JEL : L1

Classification de la Banque : Monnaie électronique 


\section{Introduction}

Even though it was introduced in 2009, the digital currency Bitcoin caught the interest of the mainstream media only in 2012. Due to its supposed anonymity, Bitcoin and other

digital currencies are often compared to cash. However, unlike cash, these currencies are purely digital and used primarily online. Digital currencies have the potential to compete against other online payment methods such as credit/debit cards and PayPal. It is possible that Bitcoin and other digital currencies may have a large long-term effect on both currency and payments systems, but these currencies are currently in their infancy. There are many unanswered questions about their viability, as well as the potential of digital currencies to be a disruptive technology.

Current developments within the Bitcoin ecosystem, as well as competition with other digital currencies, may have an important impact on the future success of this technology. We focus on decentralized digital currencies that use cryptography, called cryptocurrencies. In this paper, we analyze how network effects affect competition in the nascent cryptocurrency market. We do so by examining the changes over time in exchange rate (price) data among cryptocurrencies. Specifically, we look at two aspects: (1) competition among different currencies (Bitcoin, Litecoin, etc.), and (2) competition among exchanges where those currencies are traded. Because the supply of cryptocurrencies is either fixed or deterministically changing, changes in prices are a good indication of changes in demand.

Both in the context of currency competition and competition between exchanges, network effects play an important role. Positive network effects are present when the value of a product or service increases with the number of users. A currency is more useful as more people adopt it. An exchange is more liquid when there are more buyers and sellers. From the 'network effects' literature (cf. Katz and Shapiro 1985), in such environments we might expect a "winner-take-all" dynamics and convergence to one dominant player. The more popular the currency the more easily it can attract new users. Similarly, the larger exchange will be more attractive to new buyers and sellers. Therefore, the larger competitor will grow even larger, eventually dominating the whole market. In this paper, we ask whether the "winner-take-all" dynamics is an important force for the competition between currencies, and for the competition between the exchanges. We do not see a clear winner-take-all dynamics currently in the cryptocurrency market.

The lack of winner-take-all dynamics is less surprising for the exchanges. The nature 
of network effects is different for currency competition than for competition between the exchanges. In the exchanges, sellers benefit from a larger number of buyers, and buyers benefit from a larger number of sellers (so-called positive cross-side effects). However, sellers would prefer a lower number of other sellers, since they compete for buyers. Similarly, buyers would prefer a lower number of other buyers competing against them (so-called negative same-side effects). There are no such negative effects for currency adoption-it is always positive when more users adopt it. In the case of exchanges, the negative same-side effects may counter the "winner-take-all" dynamics (cf. Ellison and Fudenberg 2003; Halaburda and Piskorski 2011). Therefore, in an equilibrium multiple exchanges may coexist, as long as they do not provide arbitrage opportunities (i.e., neither buyers nor sellers would gain by trading at a different exchange).

The market for exchanges is very vibrant. The exchanges considered to be the "major players" changed significantly over time. New ones appeared, and existing ones were pushed out of the market. The Mt. Gox failure in February 2014 showed that even a large exchange may suddenly exit the market.

Although we have price data at only one moment a day (24:00 GMT), we examine whether there are profitable trading opportunities both within the BTC-e exchange and across the BTC-e exchange and some of the other major exchanges. We find that profitable (gross) trading opportunities are much larger across exchanges than within the BTC-e exchange. With this analysis we provide a first glimpse into trading opportunities in cryptocurrency markets. It is not a comprehensive test for arbitrage opportunities, since arbitrage opportunities can exist at any point in time, and we examine only a few of the exchanges. We will examine this issue in more detail in future research.

In competition between cryptocurrencies, we see that some of the analyzed currencies lose value and do not recover, while others keep their value for a long time. Thus, consistent with the winner-take-all dynamics, there are "winners" and "losers," and a successful currency grows more successful. But the winner-take-all dynamics is not the only force in this market, and we do not see the market tipping to one dominant currency.

Our data suggest that the winner-take-all effect is dominant only early in the market. During this period, when Bitcoin becomes more valuable against the USD, it also becomes more valuable against other cryptocurrencies. Bitcoin is the most popular cryptocurrency at the beginning of this period and during the period it further improves its position, both against the USD and against other cryptocurrencies. 
In the later period, this pattern reverses. When Bitcoin strengthens against the USD, it weakens against other top cryptocurrencies. And conversely, when it weakens against the USD, it strengthens against other top cryptocurrencies. At the end of this period, Bitcoin is stronger against the USD and weaker against other top cryptocurrencies than it was at the beginning of the period. Thus, we no longer see winner-take-all dynamics.

It has been pointed out that Bitcoin and other cryptocurrencies may be purchased as financial assets rather than for usage as currency. Both functions probably matter in the cryptocurrency market. If the main driver of demand was currency adoption, network effects would be dominant and we would see clear winner-take-all dynamics. The lack of winnertake-all dynamics in the later period indicates that the financial asset function becomes more prominent. As Bitcoin's price and volatility increase, we see a substitution effect increasing the demand for other cryptocurrencies. Thus, the prices of all the cryptocurrencies move in lockstep.

\section{Brief Background on Cryptocurrencies}

Bitcoin and the other digital currencies considered here are decentralized systems; i.e., they have no central authority. They use cryptography to control transactions, increase the supply and prevent fraud. Hence, they often are referred to as cryptocurrencies. Once confirmed, all transactions are stored digitally and recorded in a 'blockchain,' which can be thought of as an accounting system. Payments are validated by network nodes. Sometimes, as in the case of Bitcoin, powerful, expensive computers are needed for the process.

Bitcoin's algorithm provides an effective safeguard against 'counterfeiting' of the currency. However, the ecosystem is vulnerable to theft. Users keep keys to their Bitcoins and make transactions with the help of wallets. Exchanges facilitate trade between Bitcoins and fiat currencies, and also allow for storing Bitcoins. Bitcoins can be stolen through wallets or exchanges. Up until this point, exchanges have been targeted more frequently than wallets. Many wallets are located on users' computers, while exchanges by their nature are online. This makes exchanges an easier target. It was revealed in February 2014 that $\$ 350$ million worth of Bitcoins were stolen from Mt. Gox, which led to the shutdown of the exchange. ${ }^{1}$

\footnotetext{
${ }^{1}$ Wired.com, "Bitcoin Exchange Mt. Gox Goes Offline Amid Allegations of $\$ 350$ Million Hack," by Robert McMillan on 24 February 2014 (http://www.wired.com/wiredenterprise/2014/02/Bitcoins-mt-goximplodes/).
} 
The supply of most cryptocurrencies increases at a predetermined rate, and cannot be changed by any central authority. In the case of Bitcoin, in 2014 there were about 11-12 million Bitcoins in circulation, with the maximum allocation to ultimately reach 21 million.

Bitcoin was initially popular in part because its (perceived) anonymity enabled trade in illegal goods. On 2 October 2013, the U.S. government shut down the largest website involved in that activity. (In the process, the FBI received about $1.5 \%$ of all Bitcoins in circulation at the time.) Despite the U.S. government action, Bitcoin prices continued to climb, partly because the currency has a strong deflationary aspect to it, due to limited supply. There are also massive fluctuations in value, in part owing to speculation, security problems and general uncertainty as to how the industry will develop.

In our analysis, we use data from Bitcoin exchanges. Those exchanges operate as matching platforms. That is, users do not trade with the exchange. Rather, they announce limit orders to buy and sell, and the exchange matches buyers and sellers when conditions of both the buyer and the seller are met.

\section{$3 \quad$ Related Literature}

Bitcoin only very recently became a subject of research in economics. The topic has been of interest for longer in computer science. A small number of theoretical papers written by computer scientists address incentives. Eyal and Sirer (2013) show that mining is not incentive-compatible and that the so-called "selfish mining" can lead to higher revenue for miners who collude against others. The threshold for selfish mining to be profitable is lower than for double-spending attacks. Babaioff et al. (2012) argue that the current Bitcoin protocols do not provide an incentive for nodes to broadcast transactions. This is problematic, since the system is based on the assumption that there is such an incentive. Additional work in the computer science includes Christin (2013), who examines the anonymous online marketplace in cryptocurrencies. Böhme (2013) examines what can be learned from Bitcoin regarding Internet protocol adoption.

Some work on Bitcoin has been reported in legal journals as well, but there is very little in the economics literature. One of the few exceptions is the European Central Bank's (2012) report on virtual currencies. Using two examples, Bitcoin and Linden dollars, the report focuses on the impact of digital currencies on the use of fiat money. Gans and Halaburda (2013) analyze the economics of private digital currencies, but they explicitly 
focus on currencies issued by platforms such as Facebook or Amazon (that retain full control), and not decentralized currencies such as Bitcoin. This analysis is further extended in Fung and Halaburda (2014). Dwyer (2014) provides institutional details about digital currency developments. Yermack (2013) analyzes changes in Bitcoin price against fiat currencies and concludes that its volatility undermines its usefulness as currency. Moore and Christin (2013) empirically examine Bitcoin's exchange risk. Using Bitcoin traffic at Wikipedia, Glaser et al. (2014) examine whether user interest in cryptocurrencies is due to interest in a new investment asset or in the currencies themselves. Their results suggest that most of the interest is due to the investment asset function.

\section{Data}

Our analysis focuses primarily on the top currencies that have been traded for a relatively long period of time (since 2 May 2013). For consistency, we compare data for trades between currencies on the same exchange. We take advantage of the fact that some of the exchanges trade not only in Bitcoin and Litecoin but in other cryptocurrencies as well. We focus on the BTC-e exchange, ${ }^{2}$ a leading exchange that has traded several currency pairs for a relatively long time. Seven cryptocurrencies have been traded on BTC-e since 2 May 2013. They are Bitcoin (BTC), Litecoin (LTC), Peercoin (PPC), Namecoin (NMC), Feathercoin (FTC), Novacoin (NVC) and Terracoin (TRC). ${ }^{3}$ We analyze how their relative prices changed over time between May 2013 and February 2014.

We also use price data from other exchanges as well. We employ exchange Cryptsy because, as with BTC-e, it has traded the main cryptocurrencies against Bitcoin for a relatively long period of time (although for less time than BTC-e). But Cryptsy did not trade in USD, which limits some comparisons. We also employ trade data from Bitstamp and Bitfinex, since they were the largest exchanges trading Bitcoin against the USD and against Litecoin. Bitstamp and Bitfinex, however, traded only the most popular cryptocurrencies.

The source for our pricing data is http://www.cryptocoincharts.info/. This site is publicly

\footnotetext{
${ }^{2}$ BTC-e, an exchange based in Bulgaria, allows for trades in several cryptocurrencies and two fiat currencies (the USD and Russian ruble). It started trading on 7 August 2011.

${ }^{3}$ Novacoin and Feathercoin were created by "forking" Peercoin. While the top three cryptocurrencies (BTC, LTC and PPC) have been stable in terms of their market capitalization ranking, the other coins are more volatile. As of 13 January 2014, NMC was fifth, FTC twelfth, NVC fourteenth and TRC twenty-second.
} 


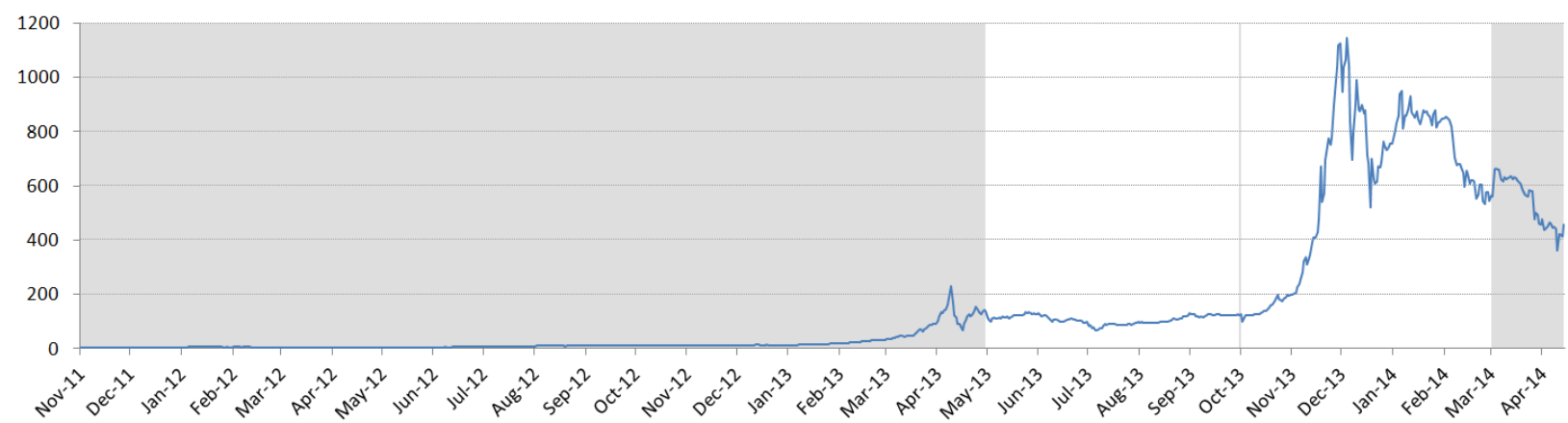

Figure 1: Bitcoin prices (in USD) over time. Shading highlights the first period (May-Sep 2013) and the second period (Oct 2013-Feb 2014) of our analysis. (Source: http://www.coindesk.com/price)

available. Trades occurring on a particular exchange are visible on its interface. The site collects and aggregates the visible data (using API, application programming interface). We use the 'closing rate,' which (from our discussions with the site manager) is the exchange rate at midnight GMT. Some days have no data for a particular trading pair or an exchange. In such cases, either the API of the exchange or something at cryptocoincharts.info was not working properly.

Casual observation of Bitcoin prices (in USD) within our data time frame, from 2 May 2013 to 28 February 2014, shows a clear difference between the price trend up to September 2013, and then from October on (see Figure 1). During the May-September period the USD/BTC exchange rate traded within a relatively narrow range (from $\$ 65 / \mathrm{BTC}$ to $\$ 130 /$ BTC), while in the period from October to February the volatility was much greater and the value of Bitcoin relative to the USD ranged from $\$ 101 / \mathrm{BTC}$ to $\$ 1076 / \mathrm{BTC}$. This price trend change may be related to the 2 October shutdown of Silk Road, a website trading in illegal substances. Additionally in October, Chinese Internet giant Baidu started accepting Bitcoin, which increased Bitcoin's popularity in China. Furthermore, in mid-November 2013, U.S. Congressional hearings cast cryptocurrencies in a relatively favorable light. For all of these reasons, this seems like a natural break in the data. ${ }^{4}$ Therefore, we examine those two periods separately. In subsequent analysis, we call the period from 2 May to 30 September 2013 the first period, and the period from 1 October 2013 to 28 February 2014

\footnotetext{
${ }^{4}$ It is not crucial that the break point occurs exactly in the middle of our data. Our results are robust to shifting the break point. We start with 2 May 2013 because we could not obtain data on all cryptocurrencies used in the analysis before this date.
} 
the second period. Table 1 summarizes the differences between the two periods.

First period $\quad$ Second period

\begin{tabular}{ccc}
\hline dates & 2 May -30 Sep 2013 & 1 Oct $2013-28$ Feb 2014 \\
major events & Silk Road raid \\
media coverage & none & Baidu accepts Bitcoin \\
UTC/USD price range & $\$ 65-130 /$ BTC & U.Songress hearings \\
& & $\$ 101-1076 /$ BTC
\end{tabular}

Table 1: First and second period in our data

\section{Competition between the Currencies}

In this section, we analyze the competition between cryptocurrencies using price data. Media coverage has mostly focused on Bitcoin. Thus, it may come as a surprise that there are around 200 cryptocurrencies. ${ }^{5}$

The other cryptocurrencies in our analysis are very similar to Bitcoin and have been created by "forking" the main Bitcoin protocol. Hence, they are called altcoins. But they are not exactly the same. For example, Litecoin will generate 4 times as many coins (84 million), and the transactions are added to the blockchain 4 times faster than Bitcoin (2.5 minutes against Bitcoin's 10 minutes). ${ }^{6}$ Peercoin relies on proof-of-stake in addition to proofof-work ${ }^{7}$ to record transactions in the blockchain (i.e., mining), thus mitigating the need for powerful, expensive computers that became necessary for mining in Bitcoin. Peercoin also does not have a limit on the total number of coins generated (although the number of coins generated at any time is known in advance).

Market capitalization values for different "coins" are quite skewed. According to http://coinmarketcap.com/, total market capitalization in digital currencies was approxi-

\footnotetext{
${ }^{5}$ See http://coinmarketcap.com/.

${ }^{6}$ There are, of course, other differences between Bitcoin and Litecoin, such as different hashing algorithms.

${ }^{7}$ For the description of technical aspects of cryptocurrencies, see http://en.bitcoin.it/wiki.
} 
mately $\$ 8.1$ billion on 26 February $2014 .^{8}$ Bitcoin accounts for approximately $90 \%$ of total digital currency market capitalization. The second-largest market capitalization is Litecoin, with approximately $5 \%$ of total digital currency market capitalization. Peercoin, the digital coin with the third-largest market capitalization value, accounts for $1 \%$ of total market capitalization. These currencies were among the early entrants into the cryptocurrency market. Bitcoin - the oldest one - was established in 2009, Litecoin in 2011 and Peercoin in 2012. Interest has grown recently, and many new cryptocurrencies entered the market in 2013 and 2014.

Many of the altcoins - especially early ones, like Litecoin and Peercoin - were developed to fix what their developers perceived as shortcomings of Bitcoin. Some of the changes may attract only a narrow group of users (e.g., Namecoin aims at more anonymity than Bitcoin), while some may have wider appeal as alternatives to Bitcoin. ${ }^{9}$ However, it has been postulated that the recent surge in entry into the digital coin market is due to the fact that, on the one hand, the entry is relatively costless, and, on the other hand, the founders of coins have made significant profits (even the coin with the 34th-largest market capitalization had a value of more than one million dollars in February 2014).

Those two motivations for the introduction of new cryptocurrencies (fixing shortcomings of Bitcoin and capitalizing on potential popularity) illustrate a disagreement about what is driving the demand for cryptocurrencies - whether people buy them due to their potential as currency, or for speculative purposes (i.e., as a financial asset). ${ }^{10}$

We argue that both forces are at work in the market, with different effects:

(1) The reinforcement effect is the result of the one-sided network effects present in the currency adoption process: As Bitcoin becomes more popular, more people would believe that it will win the "winner-take-all" race against other cryptocurrencies. With this expectation, the demand will further increase.

(2) The substitution effect is the result of speculative dynamics, i.e., treating cryptocur-

\footnotetext{
${ }^{8}$ This calculation excludes Ripple, which had a market capitalization of approximately $\$ 1.4$ billion. Ripple currency, XRP, exists (and trades) only within the Ripple system, and Ripple was designed as a currency exchange and payments system, rather than an alternative digital currency.

${ }^{9}$ It has recently been reported in the press that merchants increasingly accept Litecoin as a less-costly alternative to Bitcoin (http://www.businessweek.com/news/2014-04-24/Bitcoin-runner-up-Litecoin-emergesas-low-price-challenger-tech).

${ }^{10}$ See Yermack (2013) and Glaser et al. (2014).
} 


\begin{tabular}{cccc} 
Currency & $\begin{array}{c}\text { 2 May 2013 } \\
\text { beginning of data }\end{array}$ & 30 September 2013 & $\begin{array}{c}\text { 28 February 2014 } \\
\text { end of data }\end{array}$ \\
\hline$r(\mathrm{USD} / \mathrm{BTC})$ & 106.8 & 123.0 & 537.5 \\
$r(\mathrm{LTC} / \mathrm{BTC})$ & 31.3 & 56.6 & 40.9 \\
$r(\mathrm{PPC} / \mathrm{BTC})$ & 378.8 & 471.7 & 166.1 \\
$r(\mathrm{NMC} / \mathrm{BTC})$ & 97.8 & 246.3 & 166.7 \\
$r(\mathrm{NVC} / \mathrm{BTC})$ & 31.9 & 33.5 & 74.5 \\
$r(\mathrm{FTC} / \mathrm{BTC})$ & 197.2 & 1111.1 & 2500.0 \\
$r(\mathrm{TRC} / \mathrm{BTC})$ & 198.4 & 769.2 & 2777.8
\end{tabular}

Table 2: Exchange rates of selected currencies against BTC on BTC-e exchange

rencies as financial assets: As Bitcoin becomes more popular and more expensive, people fear that it may be overvalued (or too volatile) and look for an alternative cryptocurrency investment.

We analyze the relative prices of seven cryptocurrencies traded at BTC-e: Bitcoin (BTC), Litecoin (LTC), Peercoin (PPC), Namecoin (NMC), Feathercoin (FTC), Novacoin (NVC) and Terracoin (TRC). Since the supply of all those currencies is either fixed or deterministically changing (i.e., not adjusted in response to prices), changes in prices are a good measure of changes in demand. ${ }^{11}$

Table 2 shows exchange rates of currencies used in the analysis against BTC. The table reports the first and last day of our data (2 May 2013 and 28 February 2014), as well as the threshold date between the first and second periods (30 September 2013). Figure 2 graphically represents the changes in the exchange rates over those three dates.

We see two effects in Figure 2. First, some coins take off, while others do not. We can distinguish two groups: LTC, PPC and NMC retain their value against BTC over time (we call them 'successful'), while FTC and TRC decline significantly in value in both periods. ${ }^{12}$

Second, the value of the coins that take off increases only in the second period. In the first period, as Bitcoin becomes more valuable (against the USD), its value also increases against

\footnotetext{
${ }^{11}$ At the same time, in this case, trade volumes may be misleading as an indicator of demand. If everybody wants to buy Bitcoin, e.g., believing it will become widely adopted as currency, the volume would be low or even null, since nobody would want to sell, but the price of a trade, if any, would be high.

${ }^{12} \mathrm{NVC}$ loses quite a bit of value as well, but its decline occurs in the second period.
} 


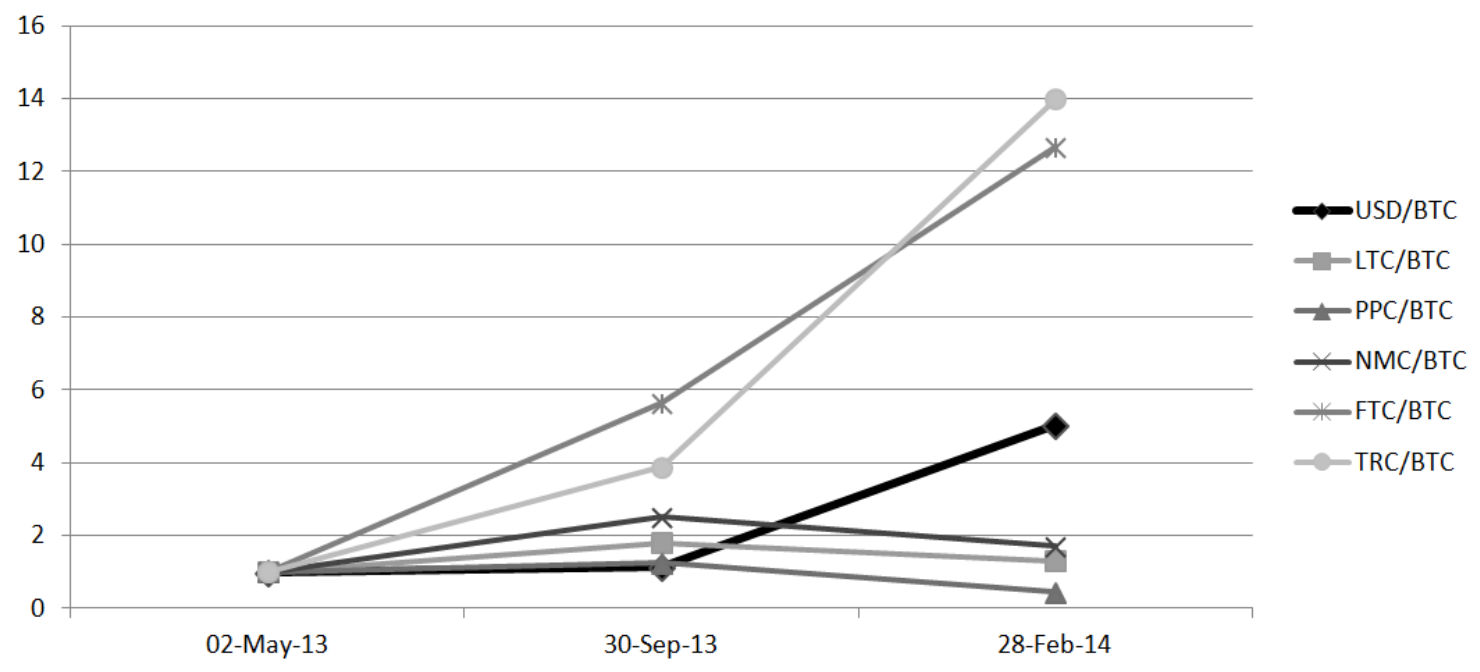

Figure 2: Changes in exchange rates of various currencies against BTC over the threshold dates in our data. Rate on 2 May 2013 is normalized to 1.

\begin{tabular}{|c|c|c|c|c|c|c|c|c|c|}
\hline & $r\left(\frac{\mathrm{USD}}{\mathrm{BTC}}\right)$ & $r\left(\frac{\mathrm{LTC}}{\mathrm{BTC}}\right)$ & $r\left(\frac{\mathrm{PPC}}{\mathrm{BTC}}\right)$ & $r\left(\frac{\mathrm{NMC}}{\mathrm{BTC}}\right)$ & & $r\left(\frac{\mathrm{USD}}{\mathrm{BTC}}\right)$ & $r\left(\frac{\mathrm{LTC}}{\mathrm{BTC}}\right)$ & $r\left(\frac{\mathrm{PPC}}{\mathrm{BTC}}\right)$ & $r\left(\frac{\mathrm{NMC}}{\mathrm{BTC}}\right)$ \\
\hline$r\left(\frac{\mathrm{USD}}{\mathrm{BTC}}\right)$ & 1.00 & & & & $r\left(\frac{\mathrm{USD}}{\mathrm{BTC}}\right)$ & 1.00 & & & \\
\hline$r\left(\frac{\mathrm{LTC}}{\mathrm{BTC}}\right)$ & 0.78 & 1.00 & & & $r\left(\frac{\mathrm{LTC}}{\mathrm{BTC}}\right)$ & -0.78 & 1.00 & & \\
\hline$r\left(\frac{\mathrm{PPC}}{\mathrm{BTC}}\right)$ & 0.09 & 0.31 & 1.00 & & $r\left(\frac{\mathrm{PPC}}{\mathrm{BTC}}\right)$ & -0.77 & 0.93 & 1.00 & \\
\hline$r\left(\frac{\mathrm{NMC}}{\mathrm{BTC}}\right)$ & 0.28 & 0.72 & 0.45 & 1.00 & $r\left(\frac{\mathrm{NMC}}{\mathrm{BTC}}\right)$ & -0.61 & 0.91 & 0.92 & 1.00 \\
\hline
\end{tabular}

(a) In first period (152 observations)

(b) In second period (150 observations)

Table 3: Correlations in daily closing prices at BTC

the other cryptocurrencies. In the second period, Bitcoin's value in USD increases further, but the values of the successful coins (LTC, PPC and NMC) increase against Bitcoin. Hence, in the second period, the values of the successful coins against the USD increase faster than Bitcoin's value.

Of course, three data points are not enough to draw conclusions. Therefore, we also examine correlations in the daily exchange rates of the successful cryptocurrencies and the USD against Bitcoin. The results are shown in Tables $3 \mathrm{a}$ and $3 \mathrm{~b} .{ }^{13}$ We use $r$ (USD/BTC) to denote the exchange rate between the USD/BTC, and similarly for other exchange rates. The

\footnotetext{
${ }^{13}$ Note that we do not include the failing currencies from Table 2 in subsequent tables. Descriptive statistics for this and other analyses are in the appendix.
} 


\begin{tabular}{|c|c|c|c|c|c|c|c|}
\hline & $r\left(\frac{\mathrm{LTC}}{\mathrm{BTC}}\right)$ & $r\left(\frac{\mathrm{PPC}}{\mathrm{BTC}}\right)$ & $r\left(\frac{\mathrm{NMC}}{\mathrm{BTC}}\right)$ & & $r\left(\frac{\mathrm{LTC}}{\mathrm{BTC}}\right)$ & $r\left(\frac{\mathrm{PPC}}{\mathrm{BTC}}\right)$ & $r\left(\frac{\mathrm{NMC}}{\mathrm{BTC}}\right)$ \\
\hline$r\left(\frac{\mathrm{LTC}}{\mathrm{BTC}}\right)$ & 1.00 & & & $r\left(\frac{\mathrm{LTC}}{\mathrm{BTC}}\right)$ & 1.00 & & \\
\hline$r\left(\frac{\mathrm{PPC}}{\mathrm{BTC}}\right)$ & 0.15 & 1.00 & & $r\left(\frac{\mathrm{PPC}}{\mathrm{BTC}}\right)$ & 0.93 & 1.00 & \\
\hline$r\left(\frac{\mathrm{NMC}}{\mathrm{BTC}}\right)$ & 0.19 & 0.32 & 1.00 & $r\left(\frac{\mathrm{NMC}}{\mathrm{BTC}}\right)$ & 0.91 & 0.91 & 1.00 \\
\hline
\end{tabular}

(a) In first period (117 observations)

(b) In second period (149 observations)

Table 4: Correlations in daily closing prices at Cryptsy

positive correlations in Table 3a show that during the first period, when Bitcoin increased in value against the USD, it also increased in value against other cryptocurrencies. However, the correlations in the first period are mostly weak. That is, the demand for one cryptocurrency was weakly affected by prices of other currencies.

In the second period, the correlations reflect the patterns observed in Figure 2. In particular, we find that there is a strong negative correlation between the exchange rate $r$ (USD/BTC) and the rates for three other major currencies ( $r$ (LTC/BTC), $r$ (PPC/BTC), and $r(\mathrm{NMC} / \mathrm{BTC}))$. This means that when Bitcoin was appreciating against the USD, it was depreciating against Litecoin and the other major cryptocurrencies. The other three digital currencies' prices move in lockstep and the correlation among them is much higher than in the first period. ${ }^{14}$

To check that this is not an artifact of one exchange, we examine the same correlation at a smaller exchange, Cryptsy. We employ Cryptsy because, like BTC-e, it has traded in the main cryptocurrencies versus BTC for a relatively long period of time. Unlike BTC-e, however, Cryptsy did not trade in USD. The correlations between cryptocurrencies' prices at Cryptsy are reported in Tables $4 \mathrm{a}$ and $4 \mathrm{~b}$, and show a similar pattern as those in Tables $3 \mathrm{a}$ and 3b: in the first period, the demand for a cryptocurrency is weakly affected by the prices of others. But in the second period, the prices of different currencies move in lockstepperhaps because higher demand for one drives higher demand for others, as we show later in Granger causality analysis.

\footnotetext{
${ }^{14}$ In the second period, Bitcoin's price (in USD) rose dramatically, peaking at more than 1,000 USD per Bitcoin in mid-December 2013. Afterwards, Bitcoin's price steadily declined - and it was worth 537.5 USD at the end of the period. When we break the second period into two subperiods, we find that the negative correlation between $r$ (USD/BTC) and, say, $r$ (LTC/BTC) is present both during the rise and the fall of Bitcoin against the USD.
} 
One interpretation of these observations is that in the first period the reinforcement effect is dominant. The demand for the most popular cryptocurrency, Bitcoin, grows even stronger, and the demand for all other currencies grows weaker. For the other relatively successful currencies, demand is not strongly correlated. During that period, Bitcoin received moderate coverage in the mainstream media, but other currencies received none. It seems reasonable to expect that at that time cryptocurrencies were acquired by enthusiasts, possibly more likely believing in their potential as currencies. It is possible that Bitcoin, as being the only one popularized by the media, also could attract some demand from people who previously were not aware of cryptocurrencies.

In the second period, the network effects drive the division between the "winners" and "losers" in the cryptocurrency market. But the observations within the successful currencies are no longer consistent with the reinforcement effect. The substitution effect dominates. In the second period, the interest in some other cryptocurrencies grows. Media coverage of Bitcoin increases significantly during that time, and some media also cover alternative cryptocurrencies. As the value of Bitcoin increases in terms of the USD during the second period, the value of the successful altcoins also increases against the USD and at a faster rate (their value rose against Bitcoin). This substitution effect may be a result of the arrival of new traders to the market, who acquire and trade the cryptocurrencies more as financial assets than for their currency potential. We argue that it would be unlikely to sustain prices of cryptocurrencies as financial assets if no one believed in their potential as currency. ${ }^{15}$ This is why very few cryptocurrencies are actively traded for a long period of time. Thus, we interpret our results as indicating that for each of the four successful cryptocurrencies there is a group of traders who believe in its future as a currency. However, once the speculation is a significant force, we can no longer deduce the relative strength of these beliefs from the relative prices.

To assess the validity of our interpretation that there is more popular interest in altcoins in the second period, we employed Google Trends. Google Trends reports on relative search volume (for searches using the Google search engine) for individual terms over time, by month. It also illustrates the relative search volumes of multiple terms. We checked for the terms 'Bitcoin' and 'Litecoin.' The results are shown in Figure 3. Google Trends does not report the absolute value of the number of searches, but the highest value in the chart is normalized to 100. Because of this normalization, a positive number of searches may show

\footnotetext{
${ }^{15}$ Although there exist models in the finance literature showing that it could be possible.
} 


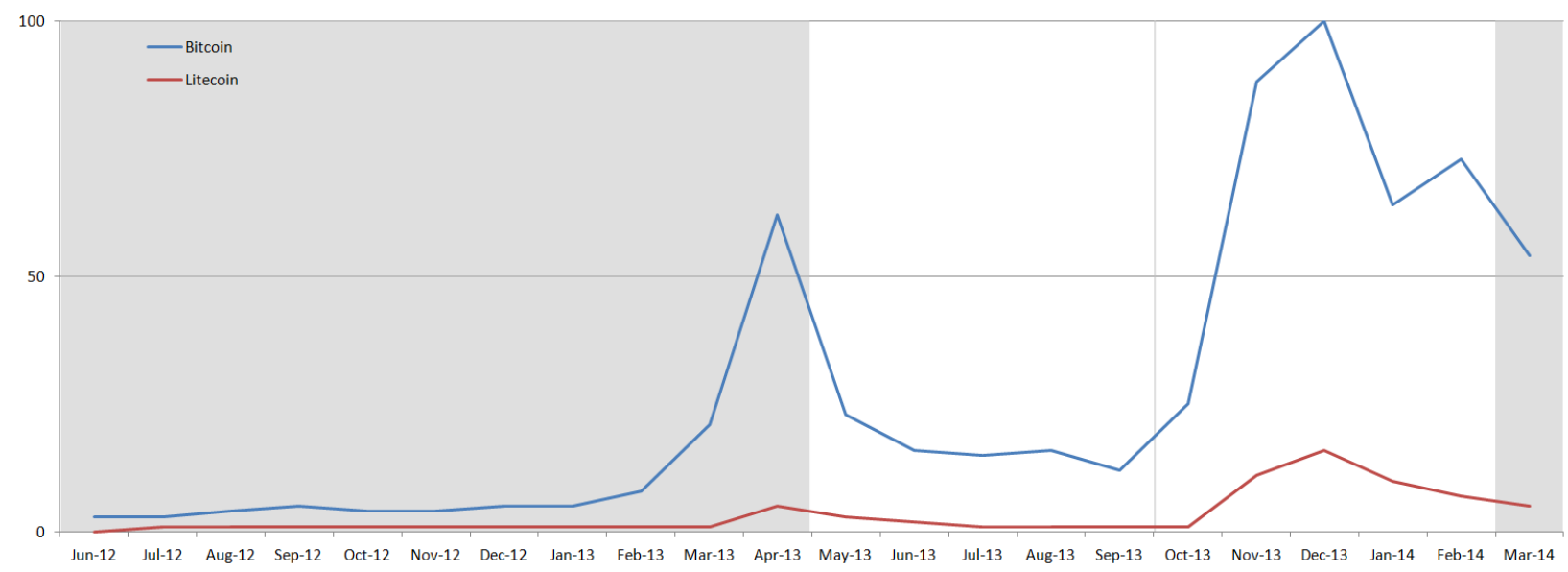

Figure 3: Google Trends results showing relative search volumes per month for terms 'Bitcoin' and 'Litecoin.' (Accessed April 2014)

up as 0 in Google Trends, if it is a sufficiently small fraction of the highest number. July 2012 is the first month in which the search volume for 'Litecoin' shows a positive number in Google Trends.

There are always fewer searches for Litecoin than for Bitcoin, but the changes over time are quite similar for the two currencies. The correlation in the number of searches between the two cryptocurrencies is 0.95. The data show that searches for both Bitcoin and Litecoin first peaked in April 2013, when the price of Bitcoin reached \$214/BTC. By 2 May 2013 (when our analysis begins), the price of Bitcoin had fallen to $\$ 106.8 /$ BTC. The Bitcoin price stays in a relatively narrow range from that point until the end of September. Similarly, the number of searches for both coins in Google Trends also stays in a very narrow range for the same period. Beginning in October 2013, the number of searches increases and reaches a second peak in December 2013. At this peak, the number for Litecoin is 16 while for Bitcoin it is 100. Bitcoin's first peak was 62, while Litecoin's was 5. Thus, the second Bitcoin peak was $61 \%$ higher than the first peak, while for Litecoin the second peak was $220 \%$ higher than the first peak. Hence, there is relatively more interest in Litecoin during the second period than during the first one.

A similar pattern is observed for Peercoin. Peercoin searches are a fraction of Litecoin searches, and an even smaller fraction of Bitcoin searches. Hence, the data are not easily visible if put on the same graph with the other two. When we look at the search intensity of Peercoin by itself over time, it also peaks in December 2013. With the reference December 
2013 as 100, Google Trends reports a positive value for Peercoin searches only from October 2013 on, which also speaks to the increased interest in alternative cryptocurrencies in the second period of our data.

In December 2013, the price of Bitcoin reaches an all-time high of $\$ 1076$. It is also the time at which Google Trends reports the most search interest for 'Bitcoin.' It is hard to say whether the price increase - covered by mainstream media-generated the search interest, or the general public interest in Bitcoin affected the demand for and price of Bitcoin. Probably both. Moreover, it seems that the increasing popularity of Bitcoin generated interest in other cryptocurrencies as well. This is why the search interest for 'Litecoin' and 'Peercoin' also surges in December 2013. It may be that the increase in price and volatility made Bitcoin more attractive as a financial asset. Seeing the large price increase, the investors interested in Bitcoin as a financial asset could be searching for alternative investment opportunities in the same category: Litecoin, Peercoin, etc.

\subsection{Granger causality analysis}

We have observed some interesting patterns in the above analysis. A natural question to ask is whether movements in the USD/BTC exchange rate 'predict' future changes in other digital currencies. Since the analysis above suggests that the first period is different from the second, we also analyze them separately here.

Given the limitations of our data, we are restricted to testing for predictability or causality in the narrow, technical sense formalized by Granger (1969) and Sims (1980). In this interpretation, a variable $x$ causes $y$ if lagged values of $x$ are significant in explaining $y$ in a regression in which lagged values of $y$ are also explanatory variables. It is, of course, possible that causality can exist in both directions. This test can be performed using vector autoregressions (VARs). We are not estimating a structural model when performing these tests; nevertheless, we believe that this type of analysis is useful in assessing whether there are differences in changes in currency movements over time. We conduct the analysis for $r(\mathrm{LTC} / \mathrm{BTC})$ and $r(\mathrm{USD} / \mathrm{BTC})$.

Not surprisingly, both of these series are non-stationary in both periods. Further, they are not cointegrated. This means that we need to conduct Granger causality tests using differenced data, that is, using the daily change in exchange rates. ${ }^{16}$

\footnotetext{
${ }^{16}$ The differenced data series are stationary. Of course, $R$-squared values are much smaller with differenced
} 
In order to conduct the analysis, we first define the following lagged variables:

$r(\mathrm{LTC} / \mathrm{BTC})(-1)=$ the lagged value of $r(\mathrm{LTC} / \mathrm{BTC})$,

$r(\mathrm{USD} / \mathrm{BTC})(-1)=$ the lagged value of $r(\mathrm{USD} / \mathrm{BTC})$.

Then the differences in the two exchange rates are defined as follows:

$$
\begin{aligned}
& \Delta r(\mathrm{LTC} / \mathrm{BTC})=r(\mathrm{LTC} / \mathrm{BTC})-r(\mathrm{LTC} / \mathrm{BTC})(-1), \\
& \Delta r(\mathrm{USD} / \mathrm{BTC})=r(\mathrm{USD} / \mathrm{BTC})-r(\mathrm{USD} / \mathrm{BTC})(-1) .
\end{aligned}
$$

We first run a regression of $\Delta r(\mathrm{LTC} / \mathrm{BTC})$ on its own lagged value and on the lagged value of $\Delta r(\mathrm{USD} / \mathrm{BTC})$. We then run a regression of $\Delta r(\mathrm{USD} / \mathrm{BTC})$ on its own lagged value and on the lagged value of $\Delta r$ (LTC/BTC). We run each of these regressions for both the first period and the second period. The results are reported in Table $5 .{ }^{17}$ We find that for the first period, neither of the lagged series is significant in explaining the daily movements of either of the two exchange rates.

In the second period, we obtain different results. In the case when $\Delta r(\mathrm{LTC} / \mathrm{BTC})$ is the dependent variable, we find that the lagged value of $\Delta r$ (USD/BTC) is significant in explaining changes in the exchange rate between Litecoin and Bitcoin. Similarly, in the case when $\Delta r$ (USD/BTC) is the dependent variable, we find that the lag of $\Delta r(\mathrm{LTC} / \mathrm{BTC})$ is significant in explaining changes in the exchange rate between the USD and Bitcoin.

We can summarize our results as follows. In the first period, neither of the lagged values predicts the current difference in the exchange rates. In the second period, the cross-lagged value of the difference predicts the current difference in the exchange rates. This indicates that, unlike period one, in period two there is two-way feedback between the difference in the exchange rates. Again, these results suggest that the first and second periods are quite different in terms of currency movements.

The Granger causality tests also suggest - like the earlier correlation data - that there is more interaction between the currency exchange rates in the second period. ${ }^{18}$ Those results

data, but the key question is whether there is feedback.

${ }^{17}$ In all tables involving regressions, $t$-statistics are in parentheses.

${ }^{18}$ When we perform the Granger analysis using PPC instead of LTC, we find no feedback in the first period and feedback from PPC/BTC to USD/BTC (but not vice versa) in the second period. Finally, when we perform the Granger analysis using NMC instead of LTC, we find no feedback at all in either period. 


$$
\begin{gathered}
\text { Dependent Variables } \\
\Delta r(\mathrm{LTC} / \mathrm{BTC}) \quad \Delta r(\mathrm{USD} / \mathrm{BTC})
\end{gathered}
$$

First period Second period First period Second period

\begin{tabular}{ccccc}
\hline Independent Variables & & & & \\
Constant & 0.17 & -0.060 & 0.024 & 3.41 \\
& $(1.25)$ & $(-0.13)$ & $(0.08)$ & $(0.78)$ \\
$\Delta r(\mathrm{LTC} / \mathrm{BTC})(-1)$ & 0.0059 & -0.12 & 0.13 & $1.88^{*}$ \\
& $(0.07)$ & $(-0.14)$ & $(0.66)$ & $(2.35)$ \\
$\Delta r(\mathrm{USD} / \mathrm{BTC})(-1)$ & -0.015 & $-0.018^{*}$ & 0.022 & -0.023 \\
& $(-0.41)$ & $(-1.98)$ & $(0.27)$ & $(-0.27)$ \\
\# of observations & 152 & 148 & 152 & 148 \\
Adjusted R-squared & -0.01 & 0.01 & -0.01 & 0.03
\end{tabular}

Table 5: Granger regressions ( $t$-values in parentheses; * indicates statistical significance at the $5 \%$ level)

are consistent with our interpretation of the driving forces in the market - that in the first period the overlap of users trading in multiple cryptocurrencies was quite small. In the second period, we see more direct competition between Bitcoin and Litecoin.

\section{Currency Exchanges}

The availability of reliable currency exchanges is critical for competition among digital currencies. The currency exchange market has been evolving rapidly over time, and it is clear that we are far from equilibrium in that market.

Mt. Gox was the dominant exchange until mid-2013. In May 2013, the FBI shut down a Wells Fargo account belonging to a Mt. Gox subsidiary, seizing $\$ 2.9$ million. This weakened the exchange, since it became difficult for U.S. customers to access it (they needed to first transfer funds to a non-U.S. account). By November 2013, Mt. Gox was still an important player, but no longer a dominant exchange. The largest Bitcoin exchange at that time was BTC China, with $35 \%$ of the trades. BTC China trades only Bitcoin against Chinese yuan (CNY). ${ }^{19}$ Mt. Gox was second, with 27\%. The third and fourth were Bitstamp, with 24\%,

\footnotetext{
${ }^{19}$ See "US has Already Ceded Dominance in Bitcoin Trading," by Jon Matonis, coindesk.com, 16 November
} 
and BTC-e, with $14 \%$ of the trades. The latter three exchanges traded Bitcoin against the USD.

The landscape in the exchange markets continued to change rapidly. As of February 2014, Mt. Gox ceased operations altogether, in large part due to a security breach - and a huge loss of Bitcoins.

During this period, a large percentage of trade occurred at two exchanges in China: BTC China and OKCoin. The only fiat currency allowed in trades in these exchanges is the Chinese yuan (CNY). The relative ranking of these exchanges by volume also changed significantly. In the Chinese market, once-dominant BTC China lost a lot of volume after the 5 December 2013 announcement by the People's Bank of China banning financial institutions from processing transactions in Bitcoin. ${ }^{20}$ In contrast, the initially small exchange OKCoin gained a lot of volume.

In the case of trades involving BTC and the USD, by mid-February 2014 there were three major exchanges: BTC-e, Bitstamp and Bitfinex. BTC-e was the first of the three to trade BTC/USD, and had about $25 \%$ of the volume for this currency pair. Bitstamp, which only trades BTC/USD, had about 50\% of the volume for this currency pair, while Bitfinex, a later entrant, had 25\% of this market. Many other exchanges were active in this currency pair (USD/BTC), but the volume traded was extremely small.

In the case of LTC/BTC and LTC/USD trades, BTC-e was the dominant exchange with about $90 \%$ and $97 \%$, respectively, of the volume of trade for these two currency pairs. In the case of LTC/BTC, five other exchanges had non-trivial trade (i.e., more than 1\%) in this currency pair. In the case of LTC/USD, only one other exchange had more than $1 \%$. For PPC/BTC, the picture is similar to LTC/BTC: BTC-e dominated with about $90 \%$ of the market. Three other exchanges had more than 1\%. In the case of NMC/BTC, BTC-e had more than $95 \%$ of the volume.

Several new exchanges have entered the market. The website http://www.cryptocoincharts.info/v2/markets/info gives daily information on digital currency exchanges and transaction volume.

Given the multiplicity of exchanges, the question arises whether the prices at the exchanges differ, and whether they allow for arbitrage opportunities. Shall we expect that 2013, available at http://www.coindesk.com/us-already-ceded-dominance-Bitcoin-trading/.

${ }^{20}$ See http://www.businessweek.com/articles/2014-03-20/btc-chinas-bobby-lee-Bitcoin-isnt-reallybanned-in-china-and-its-quickly-gaining-ground. 
in the long run there will still be multiple exchanges, or will all the trade converge to one exchange?

The argument in favor of convergence to a single exchange follows from the presence of two-sided network effects. A seller prefers to sell Bitcoin (or other cryptocurrency) in an exchange that has more buyers, since it increases the probability that the trade occurs faster and at a better price. Similarly, buyers prefer to buy Bitcoins where there are many sellers. Thus, larger exchanges create thicker, more-liquid markets. We would expect that larger exchanges (with most buyers and sellers) attract many new buyers and sellers and thus grow even larger, and more quickly, than smaller exchanges.

At the same time, due to the two-sidedness of the market, there is a negative same-side effect which may prevent tipping. While a seller prefers an exchange with more buyers, the seller also prefers to avoid the exchange with a larger number of sellers, since other sellers compete for the same buyers. Existing results in the literature suggest that if there is no arbitrage opportunity between the exchanges, it is possible for multiple exchanges to coexist in the long run, despite network effects. ${ }^{21}$

In this paper, with daily closing exchange rates (midnight GMT), we examine only arbitrage opportunities in one moment a day. We check whether the differences in prices allowed for profitable triangular trading opportunities within the BTC-e exchange, and profitable trading opportunities across the BTC-e exchange and other exchanges at this one point in time. Such analysis leaves out many other potential arbitrage opportunities, e.g., buying at midnight and selling at noon. We also do not account for the costs of making the trades, which would affect realization of the trading gains. ${ }^{22}$ Those issues will be investigated in future research. But even with our limited data, we gain insight into the convergence of prices over time both within and between exchanges.

\subsection{Trading within the BTC-e exchange}

BTC-e trades multiple currencies. Based on the pairs traded at BTC-e and the closing price inormation, we can examine whether triangular trading is profitable: that is, whether exchanging the USD to BTC, then BTC for currency $X$, and finally $X$ for the USD is

\footnotetext{
${ }^{21}$ See, for example, Ellison and Fudenberg (2003).

${ }^{22}$ These costs are especially important to note in the case of trade between exchanges, since there is cost to moving assets between the exchanges (e.g., deposit fees, withdrawal fees, transaction fees) that we do not account for.
} 
profitable at closing time. We check this for $X$ being LTC, PPC and NMC.

BTC-e allows for trades of the USD/BTC, USD/LTC and LTC/BTC. No triangular trading opportunities among these currencies implies that $r(\mathrm{LTC} / \mathrm{BTC})=\frac{r(\mathrm{USD} / \mathrm{BTC})}{r(\mathrm{USD} / \mathrm{LTC})}$. Hence, we computed the ratio of $r$ (LTC/BTC) to $\frac{r \text { (USD/BTC) }}{r(\mathrm{USD} / \mathrm{LTC})}$ for our analysis. No trading opportunities implies that this ratio should equal one exactly. As noted, we used the daily closing prices at BTC-e. We look at price differences that could allow for profitable trading opportunities, depending on the ease and cost of making the trades.

We find that the mean value of this ratio is 0.999 in the first period, and 1.000 in the second period. ${ }^{23}$ We then look at the 1st percentile and 99th percentile of the observations. In the first period, $98 \%$ of the observations fell between 0.986 and 1.013. Hence, fewer than $2 \%$ of the observations had triangular trading opportunities yielding gross returns greater than $1.4 \%$ at closing time. ${ }^{24}$ There is little difference between the periods. In the second period, $98 \%$ of the observation fell between 0.985 and 1.012 .

We follow the same procedure for Peercoin, and then for Namecoin. For those two, we examine only the second period, since there was no USD/PPC or USD/NMC trade in the first period in our data.

In the case of Peercoin, the mean value for the ratio in the second period is 1.001, and $98 \%$ of the observations for Peercoin fell between 0.978 and $1.021 .^{25}$ That is, $2 \%$ of observations allow for at least $2 \%$ of gain on triangular trading. The results are very similar for Namecoin - the mean value of the ratio is also 1.001, and $98 \%$ of observations fell between 0.970 and 1.021. ${ }^{26}$ Hence, compared with Litecoin, there were larger gross triangular trading opportunities with Peercoin and Namecoin. It is reasonable, since the volumes traded in these coins were lower than Litecoin on the BTC-e exchange. We expect more liquid markets to provide fewer triangular trading opportunities.

\subsection{Tests for trading opportunities across exchanges}

In this section, we investigate whether there are profitable opportunities from trading the same pair of currencies on two different exchanges. We examine potential trades involving USD/BTC since this is the most heavily traded currency pair by volume. Again our data are

\footnotetext{
${ }^{23}$ See Table 13 in the appendix.

${ }^{24}$ The $1.4 \%$ is computed by taking $1-0.986$ and multiplying by 100 to get the percentage.

${ }^{25}$ See Table 14 in the appendix.

${ }^{26}$ See Table 15 in the appendix.
} 
from closing time, which is midnight (24:00) GMT. Moreover, we analyze only differences in prices, without accounting for the costs of making the trades.

We first compare the exchange rate between the USD and BTC on BTC-e and Bitstamp, the largest exchange trading BTC/USD. ${ }^{27}$ Specifically, we analyze the ratio of $r$ (USD/BTC, at BTC-e) to $r$ (USD/BTC, at Bitstamp). No trading opportunities would imply this ratio to be equal to 1 .

\begin{tabular}{|c|c|c|c|}
\hline \multicolumn{2}{|l|}{ First period: } & \multicolumn{2}{|l|}{ Second period: } \\
\hline \# of observations & 104 & \# of observations & 148 \\
\hline Mean & 0.9806 & Mean & 0.9784 \\
\hline St. dev & 0.0163 & St. dev & 0.0265 \\
\hline Percentiles: & & Percentiles: & \\
\hline $1 \%$ & 0.9469 & $1 \%$ & 0.8737 \\
\hline $5 \%$ & 0.9602 & $5 \%$ & 0.9343 \\
\hline $10 \%$ & 0.9635 & $10 \%$ & 0.9497 \\
\hline $25 \%$ & 0.9694 & $25 \%$ & 0.9686 \\
\hline $50 \%$ & 0.9797 & $50 \%$ & 0.9808 \\
\hline $75 \%$ & 0.9872 & $75 \%$ & 0.9959 \\
\hline $90 \%$ & 1.0005 & $90 \%$ & 1.0036 \\
\hline $95 \%$ & 1.0074 & $95 \%$ & 1.0093 \\
\hline $99 \%$ & 1.0372 & $99 \%$ & 1.0296 \\
\hline \multirow[t]{4}{*}{ Smallest values } & 0.9304 & Smallest values & 0.8734 \\
\hline & 0.9469 & & 0.8737 \\
\hline & 0.9486 & & 0.8792 \\
\hline & 0.9587 & & 0.9043 \\
\hline \multirow[t]{4}{*}{ Largest values } & 1.0168 & Largest values & 1.0171 \\
\hline & 1.0171 & & 1.0193 \\
\hline & 1.0372 & & 1.0296 \\
\hline & 1.0377 & & 1.0379 \\
\hline
\end{tabular}

Table 6: Distribution of ratio $r$ (USD/BTC, at BTC-e) $/ r$ (USD/BTC, at Bitstamp)

\footnotetext{
${ }^{27}$ Only 104 observations for the Bitstamp exchange are available for the first period from our data source due to missing observations.
} 
We observe, however, that the ratio takes a range of values below and above 1 (see Table 6). The mean of the ratio is 0.980 in the first period and 0.978 in the second. They are not statistically different than 1. For the trading opportunity, however, it is not the average that matters, but the realized values away from 1 . In the first period, the 75 th percentage point of the distribution was below one, 0.987. That means that on most days Bitcoin was cheaper on BTC-e than on Bitstamp, and on half of the days, the difference in prices would yield more than a $2 \%$ gain. ${ }^{28}$ There were days when Bitcoin was cheaper on Bitstamp, but those are fewer. And the yield is more than $2 \%$ in this direction for only two days. $^{29}$

The standard deviation of the ratio increased from 0.016 in the first period to 0.027 in the second. This was mainly driven by a few outliers. In the first period, the 5th percentile was 0.960 . That is, for $5 \%$ of the days the prices at midnight at the two exchanges were different by more than $4 \%$. The lowest value in the first period was 0.930 . In the second period, on $10 \%$ of the days the prices were different by more than $5 \%$. And on three days the difference exceeded $10 \%$, with the lowest value of the ratio being $0.873 .{ }^{30}$

At the same time, we cannot simply conclude that there were more trading opportunities in the second period across the board. For example, the median in the second period was closer to 1 than in the first period. Rather, there were few days with significantly larger trading opportunities in the second period, while on most days those opportunities were less profitable than in the first period.

We also looked at trading opportunities for Litecoin. We compared the exchange rate between the USD and LTC on BTC-e and Bitfinex, since both trade LTC/USD. Specifically, we analyze the ratio of $r$ (USD/LTC, at BTC-e) to $r$ (USD/LTC, at Bitfinex) in the second period. ${ }^{31}$ No trading opportunities would imply that this ratio is equal to 1 . We observe, however, that the mean of the ratio was 0.987 , and the 5 th percentage point of the distribution was $0.967 .^{32}$ Hence, on several days in the second period, the closing prices differed by more than $3 \%$, suggesting relatively large gross trading opportunities on these days.

Taken together, the data suggest that gross trading opportunities were much greater

${ }^{28}$ That comes from the median slightly lower than $0.98(1-0.98=2 \%)$.

${ }^{29}$ The three largest values of the ratio are $1.038,1.037$ and 1.017 .

${ }^{30}$ The four lowest values of the ratio in the second period are 0.873, 0.874, 0.879 and 0.904.

${ }^{31}$ There are no data for USD/LTC trade at Bitfinex in the first period from our data source and there are only 104 observations available for the second period.

${ }^{32}$ See Table 16 in the appendix. 
across exchanges than within exchanges. This is consistent with remarks from a professional trader, who said "I make a significant portion of my income from conducting arbitrage between different Bitcoin exchanges. The [second half of 2013] was a very profitable time for arbitrage strategies." ${ }^{33}$

\section{Conclusions}

In this paper, we investigated the nascent market of cryptocurrencies. We primarily examined competition between different currencies, focusing on Bitcoin, Litecoin, Peercoin and Namecoin. In this environment, network effects play an important role.

We divide our data into two periods: May-September 2013 and October 2013-February 2014. In the first period, Bitcoin's price was relatively stable, while in the second period it was very volatile.

We find that in the first period, Bitcoin's popularity (as measured by exchange rates) increased against the USD and other cryptocurrencies. But in the second period, the prices of other cryptocurrencies increased even more against the USD than Bitcoin did. In our interpretation of these results, it seems that Bitcoin, via its initial popularity, "opened up" the market for cryptocurrencies in general. At the same time, Bitcoin enjoyed a first-mover advantage in an environment with network effects.

We conducted our formal analysis through the end of February 2014, using two data periods of five months in length. However, we continued to collect data for future research. Data from 1 March through the end of 31 July 2014 (an additional five months) are now available. Interestingly, even Litecoin, the second strongest cryptocurrency, fell significantly against Bitcoin in this "third" period. By the end of July 2014, the exchange rate was 77 Litecoins to a Bitcoin, nearly twice the rate as at the end of February 2014. This is despite the fact that the USD/BTC rate remained relatively unchanged from 1 March through 31 July 2014. Overall, as of 31 July 2014, Bitcoin accounted for 95\% of total digital currency market capitalization. In contrast, at the end of February 2104, Bitcoin accounted for $90 \%$ of all digital currency market capitalization.

Clearly, this third period was characterized by a significant strengthening of BTC versus

\footnotetext{
${ }^{33}$ See "Bank of Canada Research: Cryptocurrency Arbitrage Doesn't Exist," by Joon Ian Wong, coindesk.com, 13 August 2014, available at http://www.coindesk.com/bank-canada-research-cryptocurrencyarbitrage-doesnt-exist/.
} 
the other cryptocurrencies. In the long term, it is not clear whether this advantage will be sufficient to keep the dominant position. For example, Businessweek.com reported on 24 April that Litecoin is increasingly more often accepted by merchants as a lower-cost alternative to Bitcoin. ${ }^{34}$ Additional data (over time) will enable more research into competition in the cryptocurrency market and will surely bring more insights into the dynamics of this market.

\footnotetext{
${ }^{34}$ See http://www.businessweek.com/news/2014-04-24/Bitcoin-runner-up-Litecoin-emerges-as-low-pricechallenger-tech.
} 


\section{Appendix}

\section{A Descriptive Statistics}

First period:

\begin{tabular}{l|ccccc} 
Variable & Obs & Mean & St.Dev. & Min & Max \\
$r($ USD/BTC $)$ & 152 & 105.5 & 15.2 & 65.5 & 129.6 \\
$r($ LTC/BTC $)$ & 152 & 40.7 & 7.4 & 27.6 & 59.7 \\
$r($ PPC/BTC $)$ & 152 & 677.4 & 128.8 & 371.7 & 943.4 \\
$r($ NMC/BTC $)$ & 152 & 181.1 & 36.4 & 97.7 & 267.4
\end{tabular}

Second period:

\begin{tabular}{l|ccccc} 
Variable & Obs & Mean & St.Dev. & Min & Max \\
$r(\mathrm{USD} / \mathrm{BTC})$ & 150 & 579.0 & 281.9 & 101.1 & 1076.0 \\
$r(\mathrm{LTC} / \mathrm{BTC})$ & 150 & 51.2 & 23.5 & 23.8 & 117.6 \\
$r(\mathrm{PPC} / \mathrm{BTC})$ & 151 & 287.2 & 174.0 & 106.4 & 671.1 \\
$r(\mathrm{NMC} / \mathrm{BTC})$ & 151 & 239.3 & 153.7 & 88.6 & 787.4
\end{tabular}

Table 7: Exchange rates of selected currencies against BTC

First period:

\begin{tabular}{l|ccccc} 
Variable & Obs & Mean & St.Dev. & Min & Max \\
$r($ LTC/BTC $)$ & 152 & 40.7 & 7.4 & 28.0 & 59.7 \\
$\frac{r(\text { USD/BTC })}{r(\text { USD/LTC })}$ & 152 & 40.7 & 7.4 & 27.6 & 60.4
\end{tabular}

Second period:

Variable

Obs Mean St.Dev. Min Max

$r(\mathrm{LTC} / \mathrm{BTC})$

$\begin{array}{lllll}150 & 51.2 & 23.5 & 23.8 & 117.6\end{array}$

$\frac{r(\mathrm{USD} / \mathrm{BTC})}{r(\mathrm{USD} / \mathrm{LTC})}$

$\begin{array}{lllll}150 & 51.2 & 23.6 & 23.7 & 116.6\end{array}$

Table 8: Summary statistics for analysis across currency triplet LTC, BTC, USD at BTC-e 
Second period:

\begin{tabular}{l|ccccc} 
Variable & Obs & Mean & St.Dev. & Min & Max \\
$r($ PPC/BTC $)$ & 91 & 161.8 & 24.4 & 106.4 & 234.2 \\
$\frac{r(\text { USD } / \text { BTC })}{r(\text { USD/PPC })}$ & 91 & 161.7 & 24.5 & 106.3 & 232.2
\end{tabular}

Table 9: Summary statistics for analysis across currency triplet PPC, BTC, USD at BTC-e

Second period:

\begin{tabular}{l|ccccc} 
Variable & Obs & Mean & St.Dev. & Min & Max \\
$r($ NMC/BTC $)$ & 144 & 240.0 & 157.3 & 88.6 & 787.4 \\
$\frac{r(\text { USD } / \text { BTC })}{r(\text { USD } / \text { NMC })}$ & 144 & 240.1 & 158.2 & 89.1 & 793.1
\end{tabular}

Table 10: Summary statistics for analysis across currency triplet NMC, BTC, USD at BTC-e

First period:

\begin{tabular}{l|ccccc} 
Variable & Obs & Mean & St.Dev. & Min & Max \\
$r($ USD/BTC, at BTC-e) & 104 & 103.8 & 15.9 & 65.5 & 126.4 \\
$r($ USD/BTC, at Bitfinex $)$ & 104 & 105.9 & 16.5 & 66.3 & 130.7
\end{tabular}

Second period:

\begin{tabular}{l|ccccc} 
Variable & Obs & Mean & St.Dev. & Min & Max \\
$r($ USD/BTC, at BTC-e) & 148 & 582.6 & 282.1 & 101.1 & 1076.0 \\
$r($ USD/BTC, at Bitfinex $)$ & 148 & 595.0 & 288.9 & 103.9 & 1132.0
\end{tabular}

Table 11: Summary statistics for analysis of USD/BTC rate across BTC-e and Bitstamp

Second period:

\begin{tabular}{l|ccccc} 
Variable & Obs & Mean & St.Dev. & Min & Max \\
$r$ (LTC/BTC, at BTC-e) & 97 & 20.8 & 6.6 & 7.4 & 40.6 \\
$r$ (LTC/BTC, at Bitfinex $)$ & 97 & 21.1 & 6.6 & 7.7 & 41.3
\end{tabular}

Table 12: Summary statistics for analysis of USD/BTC rate across BTC-e and Bitfinex 


\section{B Summary of Distributions}

\begin{tabular}{cccc} 
First period: & \multicolumn{3}{c}{ Second period: } \\
& & & \\
\# of observations & 152 & \# of observations & 150 \\
Mean & 0.99939 & Mean & 0.9998074 \\
Std. Dev. & 0.0055147 & Std. Dev. & 0.005218 \\
Percentiles: & & Percentiles: & \\
$1 \%$ & 0.9860245 & $1 \%$ & 0.9847308 \\
$5 \%$ & 0.990053 & $5 \%$ & 0.9912314 \\
$10 \%$ & 0.9923159 & $10 \%$ & 0.9933824 \\
$25 \%$ & 0.9951707 & $25 \%$ & 0.9966723 \\
& & & \\
$50 \%$ & 0.9994248 & $50 \%$ & 1.000066 \\
& & & \\
$75 \%$ & 1.003461 & $75 \%$ & 1.003093 \\
$90 \%$ & 1.006166 & $90 \%$ & 1.006165 \\
$95 \%$ & 1.007207 & $95 \%$ & 1.007207 \\
$99 \%$ & 1.012939 & $99 \%$ & 1.012388
\end{tabular}

$\begin{array}{cccc}\text { Smallest values } & 0.9848398 & \text { Smallest values } & 0.9819901 \\ & 0.9860245 & & 0.9847308 \\ & 0.98715 & & 0.9895283 \\ & 0.9877316 & & 0.9904597 \\ \text { Largest values } & 1.008913 & \text { Largest values } & 1.010049 \\ & 1.011575 & & 1.011673 \\ & 1.012939 & & 1.012388 \\ & 1.015267 & & 1.018476\end{array}$

Table 13: Distribution of ratio $r(\mathrm{LTC} / \mathrm{BTC}) / \frac{r(\mathrm{USD} / \mathrm{BTC})}{r(\mathrm{USD} / \mathrm{LTC})}$ 


$\begin{array}{cc}\text { \# of observations } & 91 \\ \text { Mean } & 1.000602 \\ \text { Std. Dev. } & 0.0071071 \\ \text { Percentiles: } & \\ 1 \% & 0.9776574 \\ 5 \% & 0.9883524 \\ 10 \% & 0.9927711 \\ 25 \% & 0.9953269 \\ & \\ 50 \% & 1.000625 \\ & \\ 75 \% & 1.006183 \\ 90 \% & 1.008815 \\ 95 \% & 1.010891 \\ 99 \% & 1.02137\end{array}$

$\begin{array}{cc}\text { Smallest values } & 0.9776574 \\ & 0.9841475 \\ & 0.9871483 \\ & 0.9871966 \\ \text { Largest values } & 1.011718 \\ & 1.012094 \\ & 1.012504 \\ & 1.02137\end{array}$

Table 14: Distribution of ratio $r(\mathrm{PPC} / \mathrm{BTC}) / \frac{r(\mathrm{USD} / \mathrm{BTC})}{r(\mathrm{USD} / \mathrm{PPC})}$ in the second period 


$\begin{array}{cc}\text { \# of observations } & 144 \\ \text { Mean } & 1.000636 \\ \text { Std. Dev. } & 0.0084834 \\ \text { Percentiles: } & \\ 1 \% & 0.9696723 \\ 5 \% & 0.9876329 \\ 10 \% & 0.989681 \\ 25 \% & 0.997047 \\ & \\ 50 \% & 1.001448 \\ & \\ 75 \% & 1.005498 \\ 90 \% & 1.009974 \\ 95 \% & 1.011848 \\ 99 \% & 1.021259\end{array}$

$\begin{array}{cc}\text { Smallest values } & 0.9602985 \\ & 0.9696723 \\ & 0.979057 \\ & 0.9825299 \\ \text { Largest values } & 1.014756 \\ & 1.017097 \\ & 1.021259 \\ & 1.021952\end{array}$

Table 15: Distribution of ratio $r(\mathrm{NMC} / \mathrm{BTC}) / \frac{r(\mathrm{USD} / \mathrm{BTC})}{r(\mathrm{USD} / \mathrm{NMC})}$ in the second period 


$\begin{array}{cc}\text { \# of observations } & 97 \\ \text { Mean } & 0.9846464 \\ \text { Std. Dev. } & 0.0371542 \\ \text { Percentiles: } & \\ 1 \% & 0.8392107 \\ 5 \% & 0.9018628 \\ 10 \% & 0.9463614 \\ 25 \% & 0.9771627 \\ & \\ 50 \% & 0.9895921 \\ & \\ 75 \% & 1.003721 \\ 90 \% & 1.01446 \\ 95 \% & 1.023843 \\ 99 \% & 1.101175\end{array}$

$\begin{array}{cc}\text { Smallest values } & 0.8392107 \\ & 0.8472727 \\ & 0.8767327 \\ & 0.8802395 \\ \text { Largest values } & 1.027624 \\ & 1.029165 \\ & 1.045455 \\ & 1.101175\end{array}$

Table 16: Distribution of ratio $r$ (USD/LTC, at BTC-e) $/ r$ (USD/LTC, at Bitfinex) in the second period 


\section{References}

[1] Babaioff, M., S. Dobzinski, S. Oren, and A. Zohar, 2012, "On Bitcoin and Red Balloons," mimeo, available at: http://dl.acm.org/citation.cfm?id=2229022

[2] Böhme, R., 2013, "Internet Protocol Adoption: Learning from Bitcoin," in IAB Workshop on Internet Technology Adoption and Transition (ITAT)

[3] Christin, N., 2013, "Traveling the Silk Road: A Measurement Analysis of a Large Anonymous Online Marketplace," in Proceedings of the 22nd International World Wide Web Conference, Rio de Janeiro, S. 213-224

[4] Dwyer, G., 2014, "The Economics of Private Digital Currency," mimeo, available at http://brianmlucey.files.wordpress.com/2014/01/gerald-dwyereconomicsdigitalcurrency.pdf

[5] Ellison, G. and D. Fudenberg, 2003, "Knife-Edge or Plateau: When Do Market Models Tip?" Quarterly Journal of Economics, vol. 118, no. 4, pp. 12491278

[6] European Central Bank, 2012, "Virtual Currency Schemes," available at http://www.ecb.europa.eu/pub/pdf/other/virtualcurrencyschemes201210en.pdf

[7] Eyal, I., and E. Sirer, 2013, "Majority is not Enough: Bitcoin Mining is Vulnerable," available at http://i.cdn.turner.com/money/2013/images/11/04/btcProc.pdf?iid=EL

[8] Fung, B., and H. Halaburda, 2014, "Understanding Platform-Based Digital Currencies," Bank of Canada Review, Spring, pp. 12-20.

[9] Gans, J., and H. Halaburda, 2013, "Some Economics of Private Digital Currency," Bank of Canada Working Paper No. 2013-38.

[10] Glaser, F., K. Zimmermann, M. Haferkorn, M. Weber and M. Siering, 2014, "Bitcoin - Asset or Currency? Revealing Users' Hidden Intentions," available at http://papers.ssrn.com/sol3/papers.cfm?abstract_id=2425247

[11] Granger, C., 1969, "Investigating Causal Relations by Econometric Models and CrossSpectral Methods," Econometrica, 37: 424-438 
[12] Halaburda, H. and M.J. Piskorski, 2011, "Competing by Restricting Choice: The Case of Search Platforms," Harvard Business School Working Paper, No. 10-098, August 2011

[13] Katz, M. and C. Shapiro, 1985, "Network Externalities, Competition, and Compatibility," American Economic Review, vol. 75, no. 3, pp. 424440

[14] Moore, T. and N. Christin, 2013, "Beware the Middleman: Empirical Analysis of Bitcoin-Exchange Risk," in Financial Cryptography and Data Security

[15] Sims, C., 1980, "Macroeconomics and Reality," Econometrica, 48: 1-48

[16] Yermack, D., 2013, "Is Bitcoin a Real Currency?," NYU Stern School of Business, available at http://papers.ssrn.com/sol3/papers.cfm?abstract_id=2361599 